\title{
Acid treatment of platelets for removal of class I human leukocyte antigen (HLA) antigens
}

\author{
Yong Zou', Xiangfu Liu', Sihong Liao', Wenda Liu', Ying Lu' ${ }^{1}$, Xueling Tan ${ }^{1}$, Fang Li ${ }^{1}$, \\ Qing Yuan ${ }^{1}$, Guilian Liu', Zhesheng Lin ${ }^{1}$, Jiefang Li ${ }^{1}$ and Dongjun Lin ${ }^{1,2 \star}$
}

${ }^{1}$ Department of Blood Transfusion, the 3rd Hospital of Sun Yat-Sen University, Guangzhou 510630, Guangdong, China. ${ }^{2}$ Department of Hematology, the 3rd Hospital of Sun Yat-Sen University, Guangzhou 510630, Guangdong, China.

Acid elution of platelets class I human leukocyte antigen (HLA) antigens is considered as a strategy for production of HLA-eluted platelets for clinical use. In this study, we investigated the effects of acid treatment for the removal of class I HLA antigens on platelet activation, apoptosis and aggregation, and to evaluate the optimal elution condition and the feasibility of clinical application of the acid-elution technique. In vitro apheresis platelets from healthy volunteers were treated with various PH levels the citric acid solution $(\mathrm{pH}=2.0,3.0,5.0,7.0)$ at $0^{\circ} \mathrm{C}$ for $10 \mathrm{~min}$. Expression of class I HLA antigens and Pselectin (CD62P) on platelet surface were analyzed by multicolor flow cytometry. The proportion of early apoptotic platelets was detected by Annexin $\mathrm{V}$ staining. The maximum platelet aggregation rate was determined by the electrical impedance aggregometry. Results showed that the expression of platelets class IHLA antigens decreased with the reduction of $\mathrm{pH}$ value accompanied by increase of CD62P expression and early apoptosis (Annexin V expression). When compared with phosphate buffered saline (PBS) treated platelets, platelets after elution with citric acid at $\mathrm{pH} 3.0$ had significantly decreased expression of class IHLA antigens and the aggregation was not impaired, although the CD62P expression and early apoptosis were significantly increased. These results indicates that elution of platelets with citric acid at pH 3.0 can be use as an attempt to product HLA-eluted platelets for clinical use.

Key words: Platelet, human leukocyte antigen (HLA), CD62P, apoptosis, acid elution.

\section{INTRODUCTION}

Platelet infusion is a main strategy for the prevention and treatment of hemorrhage due to thrombocytopenia and/or platelet dysfunction. With the wide application of platelet products, the incidence of alloimmuninity is correspondingly increasing and may result in failure of platelet infusion. This may be mainly attributed to HLA class I antibodies, followed by platelet antigen-specific

${ }^{*}$ Corresponding author. E-mail: david200310@163.com. Tel: 020-85252389.

Abbreviations: HLA, human leukocyte antigen; FCS, forward scattering angle; PBS, phosphate buffered saline; ADP, adenosine diphosphate. antibodies (HPA). Once the platelet infusion fails, patients may face clinical and economical dilemmas. Therefore, a variety of researchers attempted to find a novel strategy for the prevention of failure of platelet infusion. In China, some researchers have prepared several kinds of solution for platelet elution (Zhang et al., 2010; Chen et al., 2007). Although, platelet elution can remove some residual leucocytes and plasma proteins and reduce the incidence of non-hemolytic febrile transfusion reaction and hypersensitive response, failure of platelet infusion still occurs in clinical practice. The presence of class IHLA antigens on the platelets is the main cause of production of HLA-I antibodies and subsequent failure of platelet infusion. Therefore, to remove of Class I HLA antigens from the platelets is a key step for the prevention of invalid platelet infusion. In the present study, incubation of 
platelets with citric acid at $\mathrm{pH} 3.0$ could effectively remove the class IHLA antigens from platelets without affecting the aggregation of platelets although the activity and apoptosis of platelets increased. Therefore, this method can be used as a strategy for prevention of platelet infusion failure and as a treatment for refractory thrombocytopenia.

\section{MATERIALS AND METHODS}

\section{Instruments and reagents}

Flow cytometer (FACSCalibur; BD), platelet aggregation meter (Chrono-Log); CD41a-APC, CD62P-PE and HLA-ABC-FITC, PE mouse IgG1 Isotype control antibodies (BD Pharmingen, USA) and Annexin V Apoptosis Detection Kit II (Keygentec) were used in the present study.

\section{Sample collection}

Six apheresis platelets samples from healthy volunteers were provided by the Guangzhou Blood Center in China. The platelet count was more than $2.5 \times 10^{11}$. The platelets were mixed by continuous vibration at $22^{\circ} \mathrm{C}$ and then $2 \sim 3 \mathrm{ml}$ of platelets were obtained for the following in vitro experiments.

\section{Preparation of citric acid solution at different $\mathrm{pH}$}

$0.2 \mathrm{~mol} / \mathrm{L} \mathrm{Na}_{2} \mathrm{HPO}_{4}$ solution and $0.1 \mathrm{~mol} / \mathrm{L}$ citric acid solution were prepared. Then, both solutions were mixed at different ratios and the $\mathrm{pH}$ was adjusted to 2.0, 3.0, 5.0 and 7.0.

\section{Acid treatment of platelets}

$500 \mu \mathrm{L}$ of platelets $\left(5 \times 10^{8}\right.$ platelets) were centrifuged at $1500 \mathrm{~g}$ for $10 \mathrm{~min}$ at room temperature and the upper plasma was removed. Then, $500 \mu \mathrm{L}$ of citric acid solution containing $1 \%$ bovine serum albumin (BSA) were added followed by incubation at $0^{\circ} \mathrm{C}$ for 10 min. After washing with Ringer's acetate solution (NRA), platelets were re-suspended in phosphate buffered saline (PBS) and then counted.

\section{Detection of expression of class IHLA antigens and P-selectin} (CD62P)

In brief, platelets $\left(1 \times 10^{6}\right)$ with or without acid treatment and those treated with PBS were subjected to flow cytometry to detect the HLA-I (HLA-ABC) and P-selectin (CD62P) on the platelets. In the controls, the CD41a-APC, IgG1-PE and IgG1- FITC mouse antihuman isotype antibodies were added. In the samples, CD41aAPC, CD62P-PE and HLA-ABC-FITC antibodies $(20 \mu \mathrm{L})$ were added followed by incubation in dark for $30 \mathrm{~min}$ at room temperature and subsequent flow cytometry.

\section{Detection of platelet apoptosis}

Platelets $\left(1 \times 10^{6}\right)$ with or without acid treatment and those treated with PBS were re-suspended in $500 \mathrm{ml}$ of Binding Buffer which was then supplemented with $1 \mu \mathrm{L}$ of Annexin V-FITC followed by incubation in dark at room temperature for $10 \mathrm{~min}$ and subsequent flow cytometry.

\section{Detection of platelet function}

The platelets before treatment and those treated with PBS or citric acid solution of different pHs were re-suspended and counted in hematology analyzer. Then, the number of platelets was adjusted to a constant and $10 \mu \mathrm{L}$ of adenosine diphosphate (ADP) $(10 \mathrm{mg} / \mathrm{mL})$ were added for induction. These platelets were subjected to detection of maximal aggregation rate (\%) in an automatic platelet aggregation meter (560CA; Chrono-log, USA).

\section{Gating and analysis}

The forward scattering angle (FCS) and lateral scattering angle (SSC) were employed to determine the cell population for detection. Then, FCS and CD41a were used for gating to determine the platelets $\left(C D 41 \mathrm{a}^{+}\right)$. Finally, the proportion of cells positive for HLA$\mathrm{ABC}$ or $\mathrm{CD} 62 \mathrm{P}$ was detected (Figure 1).

\section{Statistical analysis}

Statistical analysis was performed with SPSS version 15.0 statistic software package, and data were expressed as means \pm standard deviation $(\bar{x} \pm s)$. Comparisons of means between groups were done with student-t test and those among multiple groups with one way analysis of variance. A value of $\mathrm{P}<0.05$ was considered statistically significant.

\section{RESULTS}

\section{Expressions of class I HLA antigens and CD62P on platelets after acid treatment}

Flow cytometry showed that, when compared with platelets before treatment, the class IHLA antigens and CD62P expressions on the platelets were not markedly decreased after treatment with PBS, or citric acid solution at pH 5.0 and $7.0(\mathrm{P}>0.05)$. However, after treatment with citric acid at pH 2.0 and 3.0, the expression of HLA-I was significantly reduced from $98.8 \pm 1.5$ to $8.9 \pm 2.1 \%$ and $20.0 \pm 7.7 \%$, respectively, and that of CD62P markedly increased from $9.9 \pm 4.2$ to $84.6 \pm 8.5 \%$ and $45.6 \pm 7.9 \%$, respectively. When compared with citric acid treatment at $\mathrm{pH}$ 3.0, Expression of platelet class IHLA antigens was markedly reduced after citric acid treatment at $\mathrm{pH} 2.0$ $(P<0.05)$, and the CD62P remarkably increased $(P<0.05)$ (Figures 1, 2 and 3). The results presented are the mean \pm standard deviation. For each group, six platelet samples were analyzed. ${ }^{*} \mathrm{P}<0.05$ as comparison with other groups.

\section{Apoptosis of platelets after treatment with citric acid of different $\mathrm{pH}$}

Platelets were stained with Annexin $\mathrm{V}$ and the apoptosis of platelets was determined. When compared with 

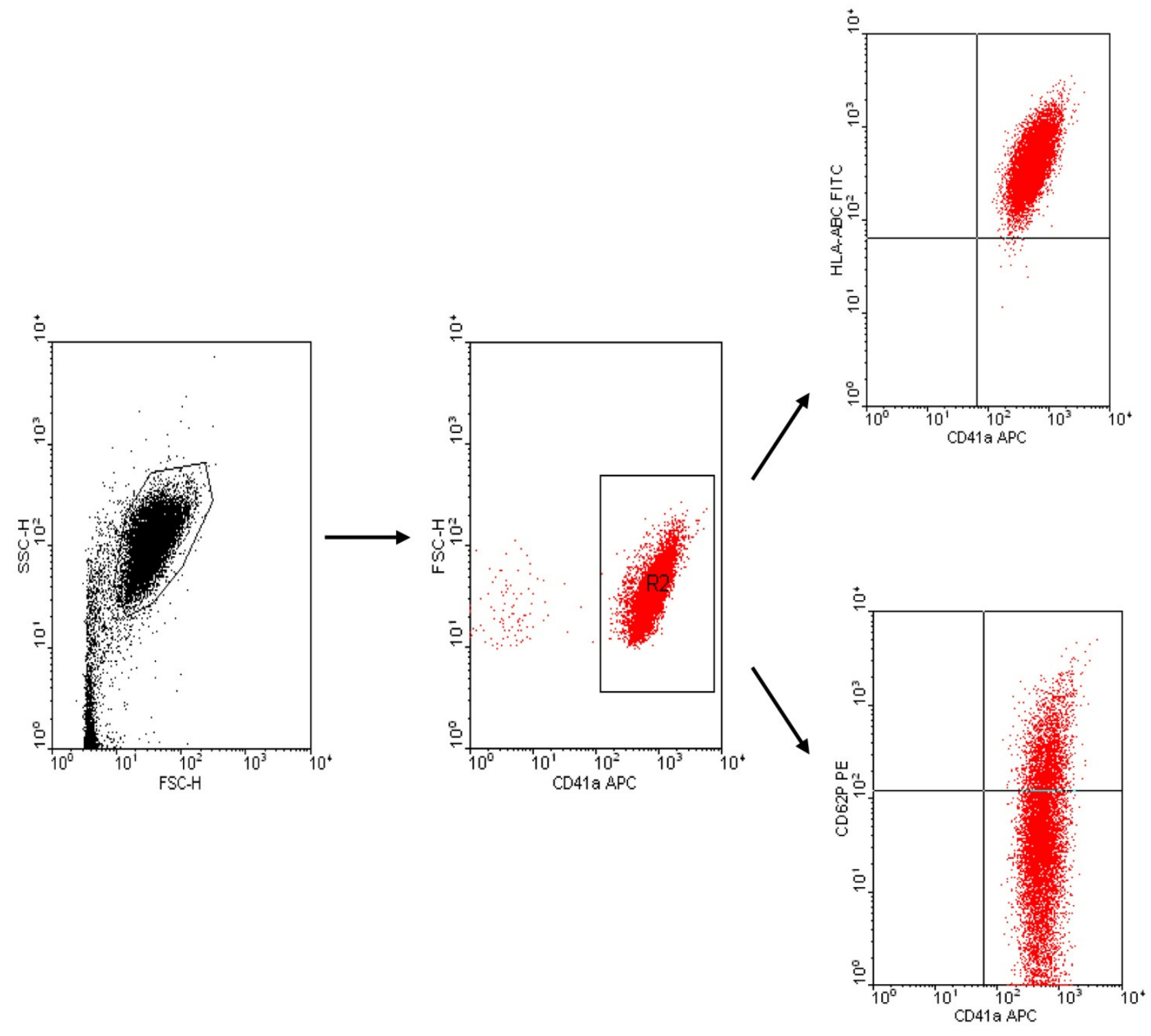

Figure 1. The schematic of multi-color flow analysis.

treatment with PBS, or with citric acid solution at $\mathrm{pH} 5.0$ or 7.0 , elution with citric acid at $\mathrm{pH} 2.0$ or 3.0 markedly increased the early apoptosis of platelets $(\mathrm{P}<0.05)$. In addition, as compared to citric acid treatment at $\mathrm{pH} 3.0$, the apoptosis was significantly increased after $\mathrm{pH}=2$ citric acid treatment $(P<0.05)$ (Figure 4). The results are presented as mean \pm standard deviation. For each group, six platelet samples were analyzed.

\section{Effects of citric acid at $\mathrm{pH} 3.0$ on aggregation of platelets}

Platelet aggregation meter was used to detect the aggregation of platelets. Results showed that when compared with aggregation before treatment, PBS or $\mathrm{pH}$
$=3.0$ citric acid treatment significantly decreased the aggregation of platelets $(P<0.05)$. Moreover, there was no marked difference in the aggregation of platelets between after PBS treatment and after $\mathrm{pH}=3.0$ citric acid treatment $(21.2 \% \pm 14.3 \%$ vs. $16.8 \% \pm 11.0 \%, P>0.05)$ (Figure 5). The results presented are the mean \pm standard deviation. For each group, six platelet samples were analyzed.

\section{DISCUSSION}

In 1989, Kurata et al. (1989) applied buffer at pH 3.0 to effectively remove class I HLA antigens on the platelets.

Their results showed the antigenicities of glycoproteins including platelet membrane glycoprotein GPIB (CD42b) and GPIlb/IIla (CD41a) were intact, the activity of 


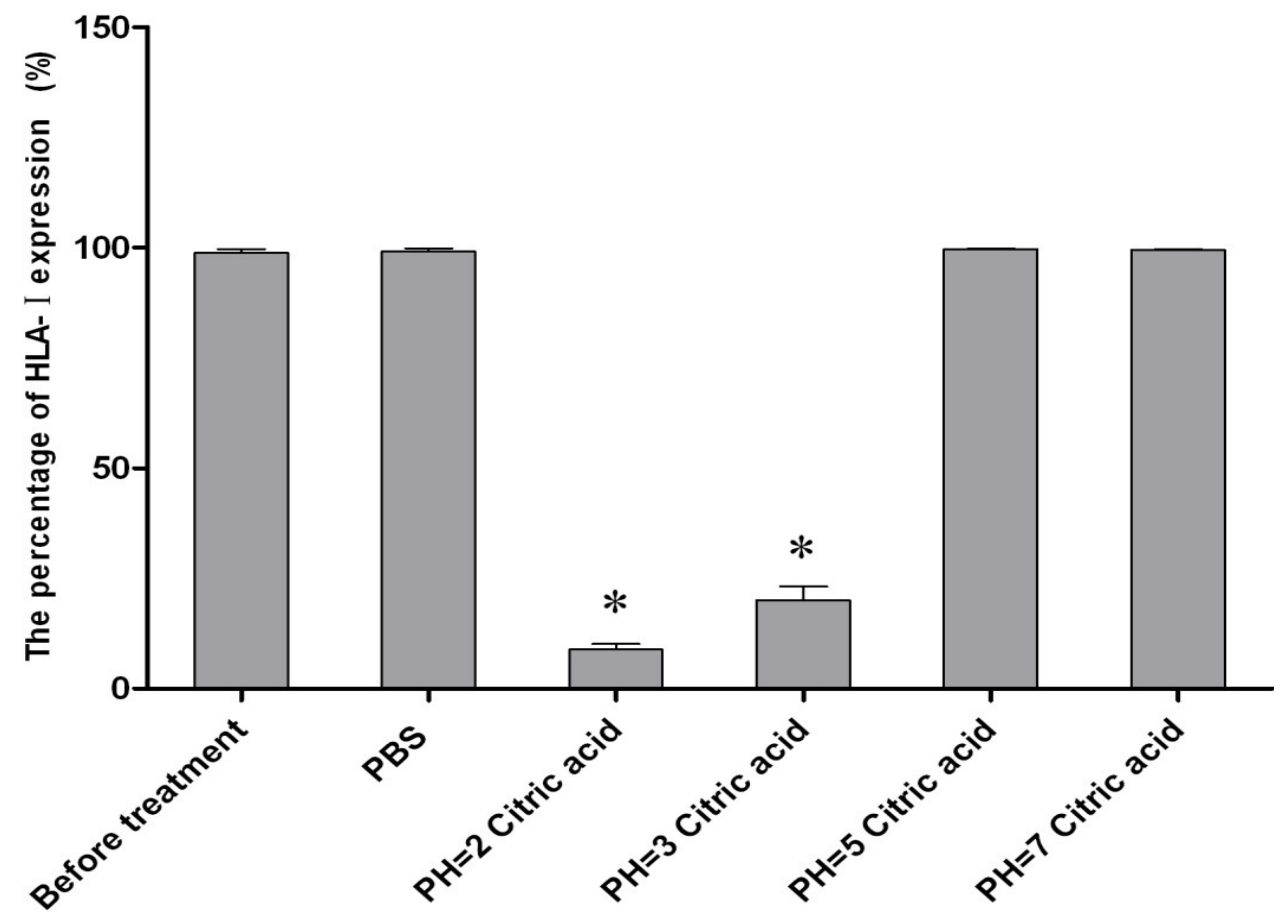

Figure 2. Expression of platelet class IHLA antigens after treatment with the citric acid solution at different $\mathrm{pH}$ levels.

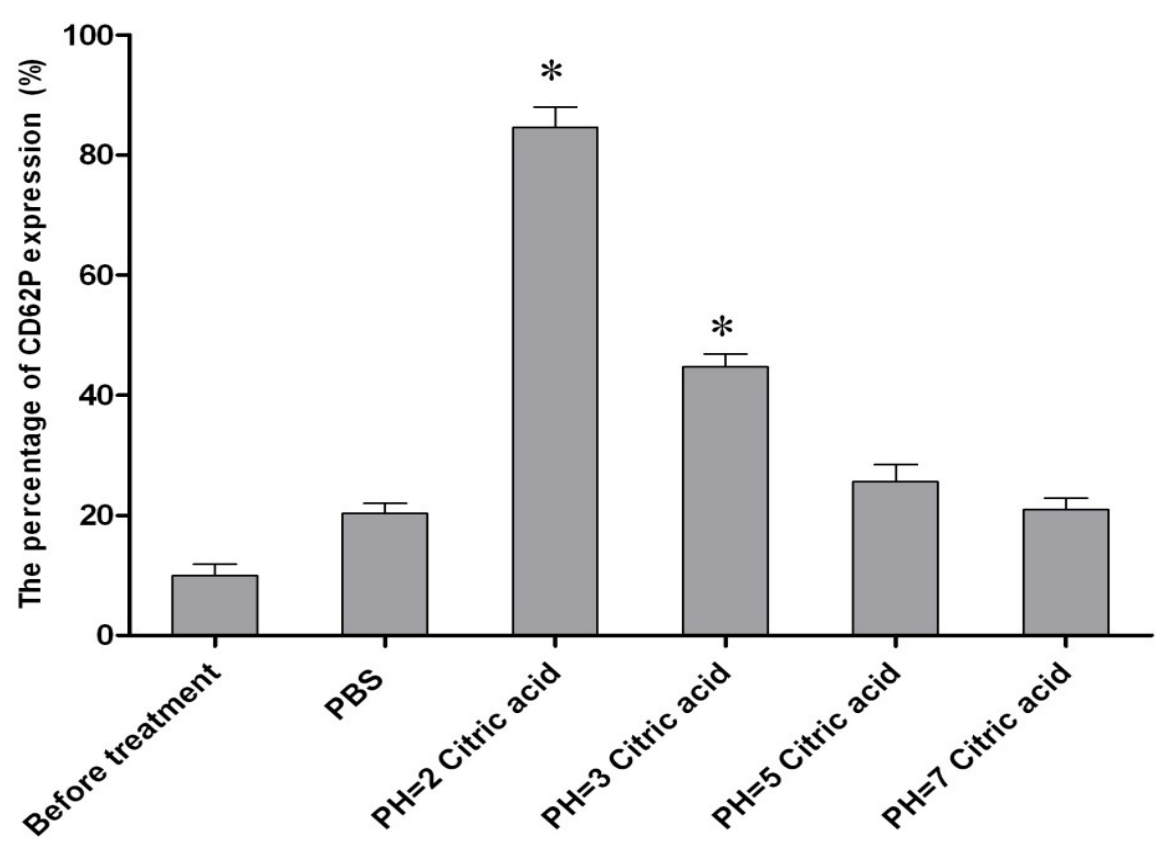

Figure 3. Expression of P-selectin (CD62P) on platelet after treatment with the citric acid solution at different $\mathrm{pH}$ levels.

platelets not markedly changed and aggregation of platelets slightly reduced. Since then, several researchers have attempted to apply acid treatment to remove class I HLA antigens on the platelets which were 


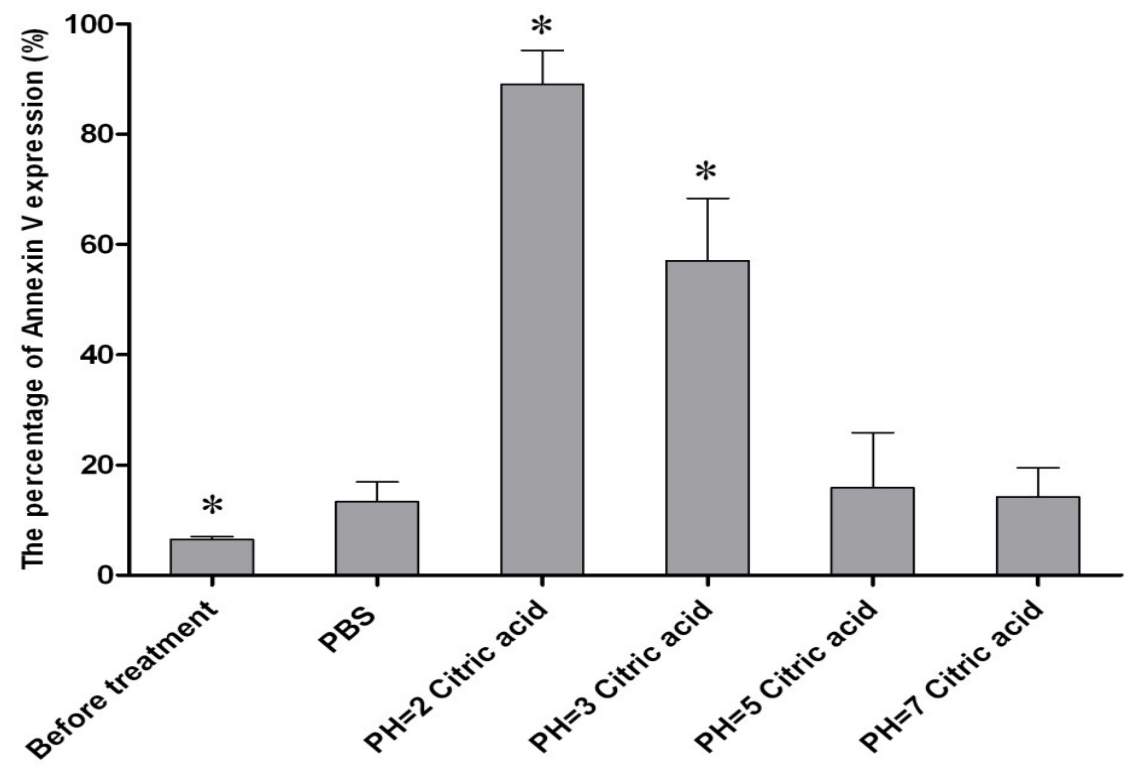

Figure 4. The proportion of early apoptotic platelets after treatment with the citric acid solution at different $\mathrm{pH}$ levels. ${ }^{*} \mathrm{P}<0.05$ as comparison with other groups.

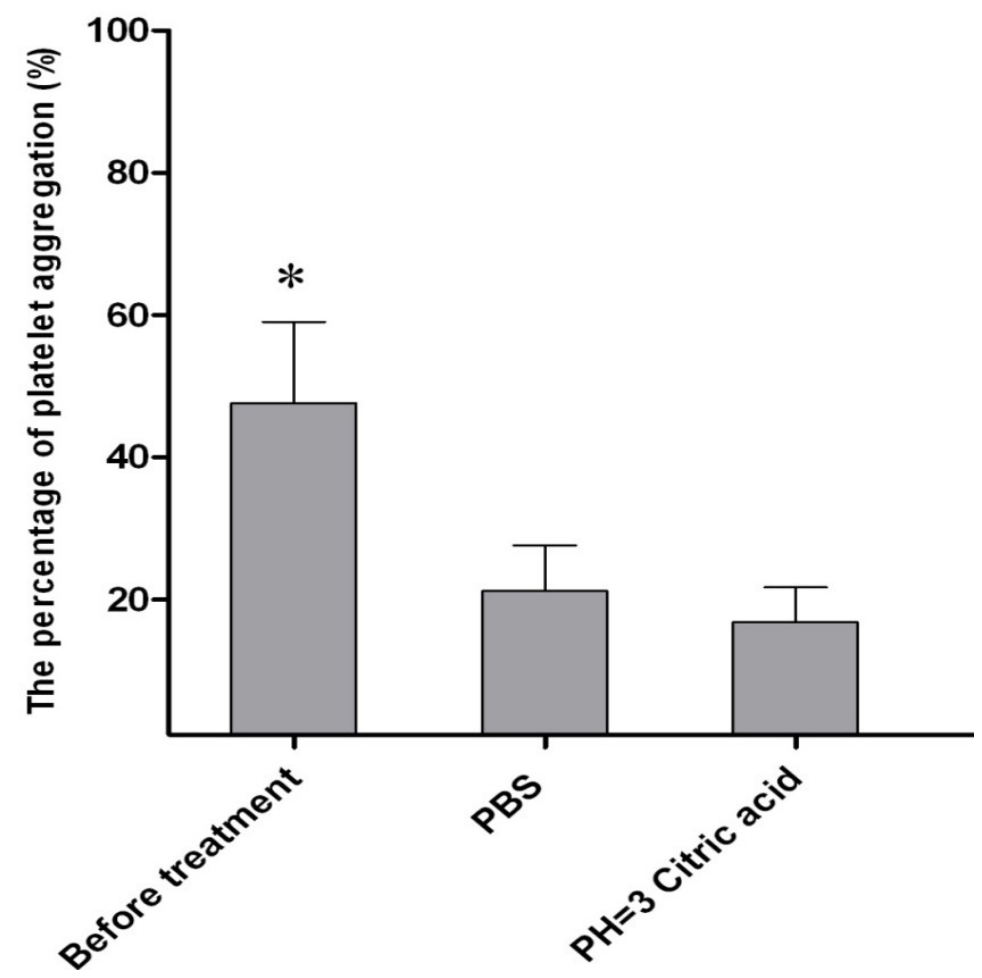

Figure 5. Effect of acid eluting class IHLA antigens with the citric acid solution at $\mathrm{pH}=3$ on platelets aggregation. ${ }^{*} \mathrm{P}<0.05$ as comparison with other groups.

then used in patients with refractory thrombocytopenia in pre-clinical studies (Novotny et al., 1996; Castro et al.,
1998). However, in 21 st century, little progression has been made in the elution of platelets with week acids in 
clinical and basic studies. To further explore the feasibility of elution of platelets with weak acid, in the present study, platelets were treated with citric acid at different $\mathrm{pH}(\mathrm{pH}=$ $2.0,3.0,5.0,7.0$ ) and the class I HLA antigens expression and apoptosis and aggregation of platelets were determined. This study reveals that:

1. The lower the $\mathrm{pH}$ value of citric acid, the higher the efficiency of removal of HLA-I on the platelets and the higher the activity and early apoptosis of platelets;

2. Treatment with citric acid at $\mathrm{pH} 3.0$ could effectively remove the HLA-I on the platelets and also significantly increased activation and early apoptosis of platelets, however, the aggregation of platelets was less affected.

Therefore, we speculate that treatment with citric acid at $\mathrm{pH} 3.0$ at $0^{\circ} \mathrm{C}$ to remove the HLA-I on the platelets can be used as a strategy for the prevention of platelet infusion failure. But the clinical feasibility of this method should be further investigated.

The increase of activity and apoptosis of platelets after acid treatment is the main obstacle in the wide application of elution of platelets with week acid. Once activated, the reserved functions of platelets are decreased and the in vivo survival rate reduced correspondingly (Holme et al., 1997; Michelson et al., 1996; Dumont et al., 2002). Our study also showed that treatment with citric acid at pH 3.0 could markedly induce the increase of the activity and apoptosis of platelets, suggesting that acid itself can damage the platelets. In spite of this, the aggregation of platelets after treatment with citric acid at $\mathrm{pH} 3.0$ was not different from that after PBS treatment. This result indicates that the platelets following acid treatment still have the function of immediate hemostasis to a certain extent. However, this strategy can not meet the clinical requirement of increasing the peripheral platelets. Therefore, new technique of acid treatment is required to develop and new inhibitors or protectors of platelet activation may protect platelets against activation during the acid treatment, which may further increase the survival of platelets in vivo. Studies in China have reported the application of reversible inhibitors of platelet activation in the platelet preservation. Liu et al. (2007) applied reversible compound inhibitors (prostaglandin

E1, L-arginine, phytate and Bivarudin of platelet activation in the platelet preservation by freeze-drying. This method decreased the CD62P expression on freezedried platelets and prolonged the survival time of these platelets. Fu et al. (2007) also found that, nitric oxide, the reversible inhibitor of platelet activation, could attenuate the activation of preserved platelets in vitro. Thus, inhibitors of platelet activation can be employed in the acid treatment of platelets. With the application of automatic blood cell washing system and new platelet washing buffer, the artificial activation of platelets will be obviously reduced. Applying this technique to remove platelet class I HLA antigens may produce platelets with high quality and safety for the clinical treatment.

\section{ACKNOWLEDGMENT}

This study was funded by the Science and Technology Program of Guangdong Province (No.2008B030301055).

\section{REFEFERENCES}

Castro E, Muncunill J, Barea L, González R, Fernández-Villalta MJ (1998). Acid elution of platelets HLA-class I antigens in the treatment of a refractory patient. Br. J. Haematol., 100(1): 245-246.

Chen HY, Yuan YG, Jiang CF (2007). The Quality of Washed Platelet Concentrates and the Outcome of Transfusion. J.Trop. Med., 7(2): 163-165(in Chinese).

Dumont LJ, AuBuchon JP, Whitley P, Herschel LH, Johnson A, McNeil D, Sawyer S, Roger JC (2002). Seven-day storage of single-donor platelets: recovery and survival in an autologous transfusion study. Transfusion, 42 (7): 847-854.

Fu YS, Zhao Y (2007). Study of the influence of nitric oxide on activation of platelets preserved in vitro. Modern. Hospital. 7(1): 68 (in Chinese).

Holme S, Sweeney JD, Sawyer S, Elfath MD (1997). The expression of $\mathrm{P}$-selectin during collection, processing, and storage of platelet concentrates: relationship to loss of in vivo viability. Transfusion. 37(1): 12-17.

Kurata Y, Oshida M, Take H, Furubayashi T, Nakao H, Tomiyama Y, Kanayama $Y$,

Nagao N, Okubo Y, Yonezawa T (1989). New approach to eliminate HLA class I antigens from platelet surface without cell damage: acid treatment at pH 3.0. Vox. Sang. 57(3): 199-204.

Liu JH, Zhou J, Wang DM, Ouyang XL, Xing YC, Lu FQ (2007). Experimental Study on Lyophilization of Platelets. J. Exp.Hematol. 15 (5): 1098-1101(in Chinese).

Michelson AD, Barnard MR, Hechtman HB, MacGregor H, Connolly RJ, Loscalzo J, Valeri CR (1996). In vivo tracking of platelets: circulating degranulated platelets rapidly lose surface P-selectin but continue to circulate and function. Proc. Natl. Acad. Sci. U.S.A, 93(21): $11877-$ 11882.

Novotny VM, Huizinga TW, van Doorn R (1996). HLA class I-eluted platelets as an alternative to HLA-matched platelets. Transfusion, 36(5): 438-444.

Zhang W, Xu GM, Liu QN (2010). Effects of different washing buffers on platelet concentrate. Chin. J. Blood. Trans., 23(11): 944-946 (in Chinese). 